\title{
Femmes, genre, migration et mobilités
}

Christine Catarino et Mirjana Morokvasic

\section{OpenEdition \\ Journals}

Édition électronique

URL : https://journals.openedition.org/remi/2534

DOI : $10.4000 /$ remi.2534

ISSN : $1777-5418$

Éditeur

Université de Poitiers

Édition imprimée

Date de publication : 29 mai 2005

Pagination : 7-27

ISBN : 2-911627-39-3

ISSN : 0765-0752

Référence électronique

Christine Catarino et Mirjana Morokvasic, «Femmes, genre, migration et mobilités », Revue européenne des migrations internationales [En ligne], vol. 21 - $\mathrm{n}^{\circ} 1$ | 2005, mis en ligne le 22 septembre 2008,

consulté le 14 avril 2022. URL : http://journals.openedition.org/remi/2534; DOI : https://doi.org/ 10.4000/remi.2534

Ce document a été généré automatiquement le 14 avril 2022.

(c) Université de Poitiers 


\title{
Femmes, genre, migration et mobilités
}

\author{
Christine Catarino et Mirjana Morokvasic
}

1 Il est des exercices incontournables : parler de la féminisation des courants migratoires en fait partie, pour toutes celles et ceux qui prétendent un jour parler des femmes en migration. Tout se passe comme si l'on devait légitimer sans cesse cet objet de recherche. Personne jamais - ou si peu - n'entame une présentation de la migration masculine en indiquant des sex ratio. Constat réitéré d'un androcentrisme qui traverse encore les sciences sociales et qui fait de l'homme le référent universel. Tout se passe également comme si tout écrit sur les femmes migrantes devait débuter par les silences, les oublis, le rappel rituel de l'invisibilité des femmes. Pourtant plus de vingt ans ont passé depuis la publication du numéro d'International Migration Review (1984) consacré aux femmes migrantes et l'un de ses articles 'Birds of passage are also women' (Morokvasic, 1984) en réponse à la métaphore utilisée par Michael Piore (1979) relative à l'immigration, entendue comme la seule mobilité d'hommes. En outre l'information collectée depuis sur les femmes en migration est loin d'être négligeable. Nombreux sont les ouvrages, numéros spéciaux de revue, modules d'enseignement etc consacrés aux femmes et aux migrations et, depuis une quinzaine d'années, à la migration et au genre. On citera notamment les ouvrages suivants: Phizacklea, 1983 ; Chant, 1992 ; Hugo, 1999 ; Kelson et Delaet, 1999 ; Anthias et Lazaridis, 2000 ; Kofman et al., 2000 ; Willis et Yeoh, 2000 ; Ehrenrheich et Hochschild, 2003 ; Morokvasic et al., 2003. Et publiés en France, quelques numéros spéciaux de revue consacrés dernièrement à ces thématiques: Migrations Société, 1997 ; REMI, 1999; Cahiers du CEDREF 2000, 2003 ; Hommes et Migrations, 2004.

\section{De femmes à genre?}

2 Pendant longtemps et bien que les femmes n'étaient pas beaucoup moins représentées que de nos jours dans les flux migratoires mondiaux - elles constituaient $47 \%$ des migrants en 1960 contre $49 \%$ en 2000 (Zlotnik, 2003) -, le genre fut ignoré, le neutre au 
masculin fut considéré comme suffisamment légitime pour représenter tous les migrants. Sortir les femmes de l'invisibilité devint l'objectif premier de celles et ceux qui souhaitaient rompre avec l'image d'une migration où seuls des hommes pouvaient être protagonistes, les femmes, elles, restaient au pays ou suivaient, subissant la migration. Dans cette imagerie, l'homme travaillait, la femme était à charge, visible seulement en tant que membre de la famille (femmesetenfants) ${ }^{1}$. Un travail de déconstruction des représentations sociales s'est imposé. Pour rendre les femmes dans la migration sociologiquement visibles encore a-t-il fallu poser le regard là où l'on ne s'attendait pas à les trouver: la sphère du travail (certains auteurs tout en voulant mettre à jour le travail des femmes, prisonniers de ces carcans, ont pu parler de "travail ambigu», Paperman et Pierrot, 1978). Rares, minoritaires, ces travaux (Morokvasic, 1976; Moulier et Silberman, 1982) n'ont pu concurrencer l'approche évolutionniste alors dominante, qui s'inscrivait dans l'opposition paradigmatique (tradition/modernité). Pour cette raison et dans un premier temps, la production de connaissances sur les femmes immigrées n'a pas " produit de la reconnaissance » et n'a pas pu renverser la vision de la migration comme majoritairement masculine. Aujourd'hui encore lorsqu'on découvre ou re-découvre les femmes en migration, on se réfère aux années dites de migration de travail présentée comme fondamentalement masculine. La présence des femmes continue à être perçue comme le résultat de l'arrêt de la migration de travail et du regroupement familial après 1974 comme le souligne à juste titre Eleonor Kofman (1999). Regard biaisé, qui tend à se poser là où les femmes étaient supposées se trouver (la maison, le cadre de la domesticité, la sphère de reproduction, le nombre élevé d'enfants faisait d'ailleurs partie des stéréotypes d'alors). Le regroupement familial masquait aussi l'entrée des femmes sur le marché du travail (Tapinos, 1990).

3 Cette phase «compensatrice » dans la recherche - axer le propos sur les femmes, les montrer là où elles n'étaient pas visibles - a eu tout du moins le mérite d'aboutir, lentement, à une sorte d'institutionnalisation: les femmes immigrées ont acquis un droit de cité dans la recherche et les politiques publiques.

4 Mais à trop vouloir focaliser le regard sur les femmes, à trop chercher à rendre visible la face cachée de la migration, cette littérature a oublié les hommes. L'occasion de saisir le genre, la différence entre les sexes a été délaissée. Ce constat vaut également d'ailleurs pour nombre de recherches féministes, et commence à être admis, notamment dans les études relatives au travail :

"L'introduction de la différence des sexes constitue un vrai tournant: en passant des femmes au genre ou au sexe, les recherches ont changé de cap. Le reproche que l'on adresse rituellement aux sciences de l'homme peut tout aussi bien être retourné vers les «recherches féministes »: oublier les femmes et le féminin, ce n'est pas bien, mais faire l'impasse sur les hommes et le masculin, ce n'est pas franchement mieux. Or, longtemps, trop longtemps là aussi, on a omis de regarder les hommes, leurs métiers et leurs activités comme du travail masculin » (Laufer, Marry, Maruani, 2003 : 13).

5 À regarder les travaux consacrés au couple genre et migration, il apparaît pourtant que certains se limitent à une étude des femmes tout en défendant qu'une telle approche n'est pas incompatible avec la prise en compte des rapports de domination entre les sexes (Parreñas, 2001). Il est vrai que pour certain(e)s tant que les traités de sociologie des migrations, les états des lieux ou rapports d'envergure ayant prétention à englober l'ensemble des phénomènes migratoires (Commissariat Général du Plan, 2002) 
continuent d'ignorer les femmes et $a$ fortiori le genre, la phase compensatrice s'avèrera nécessaire (Morokvasic, 2003). D'autres auteurs proposent une comparaison de l'expérience migratoire des hommes et des femmes en migration (Pessar, 1999 ; Yeoh et Huang, 2003). Le débat reste entier, signalons, à ce propos, que ces deux perspectives, sont représentées dans ce volume.

6 Il est en outre usuel, s'agissant des femmes immigrées, de largement se référer ou étudier quelques secteurs d'activité présentés comme traditionnellement investis par elles: service domestique, emplois du care, prostitution et traite des femmes. La division sexuelle et ethnique du travail à l'échelle internationale les confine ainsi dans des secteurs d'activité socialement dévalorisés, considérés peu qualifiés et tenus pour une extension des activités réalisées par les femmes dans la sphère privée. Nous assistons de fait à un transfert international du travail de reproduction sociale (Truong, 1996) voyant des femmes des pays nantis exercer une activité professionnelle en se déchargeant des tâches domestiques ou assimilées sur d'autres femmes (immigrées ou femmes de minorités ethniques). Si le phénomène n'est pas nouveau, des auteurs mettent l'accent sur le développement du transfert international du care, de l'affection/ affect prodigué(e) par les "nannies", "sex workers », etc. (Ehrenrheich et Hochschild, 2003). De par le monde des femmes migrent pour occuper des emplois domestiques ou de care en laissant leur(s) propre(s) enfants aux soins d'autres femmes (de la parenté ou des personnes recrutées à cet effet). Le transfert (international) du travail de reproduction se répercute ainsi en chaîne, ce qu'Hochschild (2000) a nommé le global care chain. Si ce confinement des femmes immigrées dans ces emplois a une réalité statistique, il n'en demeure pas moins - et des auteurs le font remarquer - que l'existence d'un faible nombre de travaux relatifs aux femmes migrantes qualifiées ou investissant d'autres secteurs d'activité considérés comme valorisants (entrepreneuriat, etc.) contribue à en livrer une image victimisante.

7 Les textes de ce numéro s'inscrivent dans la continuité de ce renouvellement de production des connaissances relative aux femmes en migration d'abord, au genre et migration ensuite. Sans avoir l'ambition d'effectuer un état des lieux de la recherche sur la question, nous avons cherché à mettre les différents articles en perspective, à les confronter lorsque cela était possible, mais aussi à pointer de nouvelles pistes de recherches et éclairer telle ou telle problématique à partir d'une lecture sexuée des migrations.

Nous disposons ici de quelques axes de comparaisons forts : il est possible de confronter l'expérience de personnes issues d'un même " corps de métier " (les infirmières) chez deux groupes différents, les Philippines et les Indiennes, dans des pays ou contextes d'accueil très différents : le premier aux États-Unis, pays traditionnel d'immigration, l'autre dans les pays du Golfe qui, quant à eux, ne cherchent pas à favoriser "l'intégration » des immigrés (voir respectivement les contributions de Yen Le Espiritu et de Marie Percot); de rapprocher des contributions traitant du même groupe ethnique mais appartenant à des classes sociales différentes ou ayant des capitaux scolaires et des niveaux de qualification différents et ce, dans ou entre divers pays (les Roumains hautement qualifiés installés au Canada chez Mihaela Nedelcu; les Roumains moins qualifiés circulant entre la Roumanie et respectivement la France, l'Espagne et l'Angleterre chez Swanie Potot). Les autres axes de comparaison, moins évidents sont multiples. Les contributions présentées couvrent également un large spectre thématique et géographique: réorientations et circulations migratoires, 
activités commerciales, traite des femmes, mobilité sociale, lobbying et mobilisations collectives, stéréotypes et représentations sociales, contractions d'unions mixtes, rapports sociaux ou (re) configuration des rapports sociaux de sexe au sein des familles; le tout, de la Californie à la France en passant par les pays du Golfe. Les actrices de ces migrations sont Philippines, Indiennes, Roumaines, Polonaises, Espagnoles, Maghrébines, etc. ou de ces origines. Nous avons également souhaité croiser une multiplicité de regards, faisant appel à des auteurs se réclamant de disciplines différentes même si le nombre de sociologues prime. La diversité des méthodes d'enquête et de recherche est également notable: suivi de migrants dans leurs pérégrinations, entretiens qualitatifs individuels ou en groupes, observations participantes, fréquentations des cercles étudiés (conférences, séminaires, réunions associatives, etc.), travail de dépouillement des archives et des documents issus de la « littérature grise ».

\section{Les différentes figures du transnational}

9 À l'ère de la globalisation, le «transnational » (Glick-Schiller, 1992, 2001 Portes, 1999) est devenu une référence centrale de la recherche anglo-saxonne. En Europe et en France, d'autres concepts sont privilégiés (circulation, territoires circulatoires) et rendent finalement compte d'un spectre de situations et d'expériences plus vastes que l'immigrant transnationalism (Tarrius, 1992, 1996 ; Dorai, Hily et Ma Mung, 1998 ; Péraldi, 2001).

10 La valeur heuristique que l'on doit reconnaître au concept de transnationalismeest d'avoir su capter ou saisir la complexité des phénomènes liés à la migration et à l'expérience post-migratoire dans un monde caractérisé par l'expansion des moyens de locomotion à des coûts minorés, par l'interdépendance accrue, la connexion médiatique de la planète, ainsi que l'instantanéité et la proximité virtuelle qui en résulte. Il a permis d'impulser une vision dynamique qui défie la vision statique des migrations comme mouvement «entre deux sédentarités " (Tarrius, 1992). Ce concept va à l'encontre du modèle linéaire qui suppose le mouvement migratoire comme unidirectionnel dans un espace bipolaire, impliquant les dichotomies départ-arrivée, installation-retour, temporaire-permanent, etc. Il rend compte du brouillage des schémas migratoires. Les individus franchissent ou se jouent des frontières, qui pour travailler, qui pour se soigner, se marier, étudier, etc., maintiennent des liens qui les transgressent, circulent entre elles, à travers elles, construisent leur identité en référence à plusieurs espaces nationaux. Si l'usage du terme «transnational » (tout du moins dans la littérature anglo-saxonne) semble privilégier l'acception "à travers ", c'est-à-dire la mise en relation des espaces, les mobilités et circuits franchissant les frontières, etc., il revient - paradoxalement - à mettre en avant le «national» au détriment d'ailleurs d'autres appartenances comme la classe, le genre, etc. Mais qu'en est-il de l'acception du "au-delà " (trans)national, au sens de "transgresser le national », tout aussi théoriquement possible et où les liens mis en place ou modalités d'identification ne sont pas forcément nationaux ? Quoi qu'il en soit, au brouillage ou à l'opacité des modes de circulation répond aussi celui des identités, identités multiples, hybridité(s).

11 Certains auteurs (Kivisto, 2001: Morokvasic, 2003) ont reproché à cette conceptualisation « nouvelle » d'avoir mis l'accent sur l'adaptation ou l'intégration à la 
société ambiante, en l'occurrence à la société de destination En effet l'accent mis sur les migrations d'installation et sur la durée a pu occulter d'autres formes migratoires. On n'a ainsi pas su voir comme transnationales les stratégies de celles et de ceux qui ont migré sans pour autant pouvoir ou vouloir s'installer, devenir résidents, s'adapter à la société de destination. Des situations où les individus sont mobiles, circulent, « migrent afin de rester chez eux", s'installent dans la mobilité (Morokvasic, 1992, 1999), construisent des liens transnationaux non réductibles à leur communauté d'origine et pas nécessairement durables. Les migrations pendulaires faites de va-et-vient entre la Pologne et Berlin par exemple constituent un exemple, parmi d'autres, de la façon dont des individus mettent à profit les imperfections de la législation et du marché, profitent de la perméabilité des frontières, utilisent au bout du compte leur mobilité comme ressource. C'est à ce type de mobilité que se réfèrent les contributions de Swanie Potot et de Camille Schmoll qui portent respectivement sur les Roumains - hommes et femmes - temporairement insérés à l'étranger dans diverses activités économiques (vente de journaux à Nice, agriculture en Espagne, et emplois déqualifiés du tourisme en Angleterre) et les commerçantes tunisiennes circulant entre Sousse et Naples. Migrant(e) s qui ne s'installent pas dans les pays de destination où ils (elles) exercent une activité lucrative, qui finalement migrent pour ne pas partir, dont l'ancrage demeure au pays d'origine.

12 Appréhender les circulations migratoires, demande un suivi dans le temps et dans l'espace comme le proposent les textes relatifs aux migrants roumains hommes et femmes et aux commerçantes tunisiennes entre la Tunisie et l'Italie. S'il existe désormais maints travaux portant sur la circulation impliquant les migrants postcommunistes des pays de l'Est et ceux issus ou qui essaiment le bassin euroméditerranéen (Morokvasic, 1992, 1999 ; Michalon, 2003; Hily et Ma Mung 2002 ; de Tapia, 2002), notons toutefois que leur mise en perspective reste rare. La circulation des commerçantes tunisiennes s'inscrit dans une tendance forte : celle de la féminisation de flux bien spécifiques, la migration de femmes partant seules qu'elles soient ou non mariées et provenant d'espaces où cette mobilité féminine n'était pas auparavant répandue. Et ce contrairement aux circulations ou migrations de personnes d'Europe de l'Est ou des Philippines au sein desquelles les femmes sont nombreuses, voire prédominent. De surcroît, et à la différence des femmes occupant des emplois de services, qu'elles soient infirmières, gardes d'enfants, domestiques, etc., la mobilité géographique des commerçantes tunisiennes ne répond pas à une demande de travail émanant des sociétés européennes.

13 Tout semble à première vue distinguer les commerçantes tunisiennes des circulantes Roumaines ici présentées : l'état des rapports sociaux de sexes dans les pays d'origine, le rapport des femmes à la sphère publique et au travail, etc. Dans les deux cas, ces femmes, cependant, négocient, avec leur famille, leur mobilité avant leur départ. Paradoxalement, les Tunisiennes semblent pouvoir davantage capitaliser les acquis de la migration que les Roumaines, ayant pourtant en principe bénéficié de l'égalité institutionnalisée du système communiste. La circulation et le négoce des premières sont couronnés par la possibilité d'étendre leurs activités et d'investir en Tunisie ;les secondes, en revanche, se voient coupées, entre deux migrations, des informations et opportunités économiques promues par les réseaux - masculins - liés à l'économie informelle en activité en Roumanie. 
14 Pour mieux circuler, les «pendulaires » de l'Est font usage de diverses ruses et savoirfaire sexués : l'art de s'habiller en couches, d'y enfouir bijoux et argent, de chercher à passer inaperçueset, toujours, de susciter la compassion (femmes flanquées de leur(s) enfant(s) pour voyager) (Morokvasic, 2004). Au sein des groupes mixtes de voyageurs, les femmes se verront confier ou déléguer l'épineuse négociation avec les douaniers, la gestion des interactions avec ces représentants de l'ordre, les gardiens symboliques des frontières des États qu'il leur faut franchir, tant il est admis, pour paraphraser Irek (1998) qu'on ne saurait jeter une femme d'un train. Les femmes commerçantes tunisiennes, elles, jouent sur leurs attributs de féminité pour mettre dans l'embarras les douaniers ou se servent, à leur avantage, du port du voile pour se protéger du regard et de la convoitise des hommes. Tactiques, victoires de l'instant. Et Camille Schmoll est fondée à évoquer l'art de la métis de Michel de Certeau (1990) ainsi qu'Alain Tarrius et ses «savoir-circuler» (Tarrius, 1992). On trouve donc, chez ces commerçantes tunisiennes comme chez les femmes de l'Est, un «savoir-circuler» féminin: dans les deux cas, outre que ces femmes instrumentalisent leurs attributs sexués, elles retournent à leur avantage la dissymétrie des rapports entre les sexes.

15 L'engagement, la pratique associative et politique ainsi que les revendications des migrants seraient également genrés. Des travaux menés aux États-Unis montrent que les hommes sont plus portés à s'engager dans des activités transnationales, liées à leur pays d'origine, leur permettant ainsi d'y exercer leur citoyenneté et d'y investir économiquement tandis que les femmes s'orientent plus volontiers vers les activités locales, entièrement destinées à améliorer leur sort et leur condition sociale et civique dans le pays d'accueil (Pessar 2001). Au caractère transnational et mobile de l'activité des uns est ainsi opposé l'aspect local et sédentaire de l'activité des autres. L'opposition est confirmée par la différence en matière d'orientation migratoire : les hommes sont plus enclins à vouloir retourner dans le pays d'origine tandis que les femmes concentrent leur énergie à chercher à s'installer dans le pays d'accueil. Et l'on retrouve les classiques dualités reposant sur les caractéristiques habituellement attribuées à chacun des sexes: mobilité côté masculin/enracinement côté féminin, extérieur/ intérieur, etc. Cette opposition témoigne de rapports sociaux dissymétriques car les femmes sont ainsi (réputées) exclues de l'arène publique en y exerçant, dans une moindre mesure que les hommes, leur citoyenneté et leur pouvoir économique. Sous couvert de considérer, en outre, les réseaux transnationaux comme une ressource, les femmes se trouvent donc, dans cette perspective, désavantagées.

Or, la contribution d'Helen Schwenken montre, à l'inverse, comment les employés domestiques immigrés à majorité féminine s'organisent sur une base transnationale en vue de défendre leurs droits. Si effectivement l'objectif de ces femmes consiste notamment à améliorer leur condition (de travail, leur statut juridique, etc.) dans le pays d'accueil, l'assise de leur modalité d'organisationet l'étendue de leur revendication n'en demeurent pas moins transnationales. Leur organisation et la défense des droits qu'elle porte ne se limite pas à une seule communauté d'origine, le réseau RESPECT décrit par Schwenken est interethnique, regroupe des femmes (et des hommes) de diverses origines nationales dans divers pays. Il construit en outre des alliances avec les féministes et d'autres corps de professions (les organisations syndicales) (le velvet triangle) et se présente donc comme trans-national (traversant/ transgressant les frontières nationales, de classe et de sexe). L'exemple analysé par l'auteure suggère donc que les femmes peuvent recourir aux ressources transnationales 
afin d'améliorer leur condition dans le pays d'accueil et, conjointement, dans d'autres pays. Dans la même logique, différents exemples de coalitions similaires sont rapportés par Annie Phizacklea (1998) pour qui les alliances nouées entre le transnational et le local, le communautaire et au-delà tendent vers des « transformative politics ».

Les recherches qui intègrent une perspective de genre à l'approche transnationale, de plus en plus nombreuses, viennent rendre compte des limites ou des coûts sociaux des pratiques transnationales pourtant présentées comme "alternative avantageuse » (Portes, Guarnizo, Landolt, 1999). Bémol à l'apologie du paradigme transnational, ces études se sont centrées sur les stratégies des mères transnationales ('transnational motherhood') : comment donc être mère à distance, parfois à des milliers de kilomètres de la maison et de ses enfants? Et comment le rester en dépit de la séparation qui peut perdurer toute une vie ? Finalement comment ce sentiment de maternité se modifie-til, est-il renégocié pour s'adapter à la séparation? (Hondagneu-Sotelo et Avila, 1997). Comment la notion de chez soi ('home') se construit-elle et évolue-t-elle? Des liens transnationaux familiaux accentuent-ils la rupture ou permettent-ils d'établir la continuité entre deux maisons et deux mondes (Willis et Yeoh, 2000)?

Les travaux traitant plus particulièrement du transnational motherhood, ou de la nécessité pour les femmes d'accomplir à distance les fonctions de care auxquelles les rapports sociaux de sexe les affectent en priorité, bien qu'ayant ouvert de stimulantes perspectives de recherche, ont finalement contribué à cantonner les femmes dans ladite sphère reproductive ou ont pointé leur regard vers l'articulation sphère productive/reproductive. Ils contribuent ainsi à enfermer les femmes migrantes dans le rôle de «mères nourricières ». Il s'avère difficile de les « extirper » de la famille et des relations familiales, de les penser même, ainsi que leurs expériences, autrement que dans le rapport reproduction/production. Constat allant à l'encontre de l'extension du phénomène des femmes chefs de famille de facto ou de jure, parfois principales pourvoyeuses économiques de leur foyer (transnational ou non). Travailler à l'extérieur et subvenir aux besoins de la famille est alors incorporé dans la norme, il s'agit en quelque sorte d'une extension du rôle de "bonne mère " comme l'a suggéré il y a longtemps Myra Marx Feree (1979), tandis que - paradoxalement - et comme le note avec justesse Chiho Ogaya (2004) son absence même du foyer la fait qualifier en même temps de "mauvaise mère ». Le reproche ne pèse que sur les femmes. Plus souvent posée au féminin, la question de la paternité transnationale (Pribilsky, 2000, 2004) fait figure de parent pauvre de ce type de recherche. Le "coût social» qui résulte de l'absence, de l'éloignement physique, émotionnel n'est calculé qu'au féminin. La séparation des pères d'avec les enfants n'est pas pensée comme véritablement problématique autrement que sous l'angle du soutien financier accordé à la progéniture : le père migrant enverra-t-il ou non des transferts monétaires au bénéfice du foyer et de quel montant? Résidu de l'assignation prioritaire, toujours vivace dans les esprits, des femmes aux travaux de reproduction sociale (et de care) et des hommes à la sphère du travail. Pour réduire ce sentiment de culpabilité, certaines femmes latino-américaines vivant aux États-Unis, comme l'ont indiqué Hondagneu-Sotelo et Avila (1997) ont quelque peu modifié le sens conféré à la maternité qui tend à glisser du «caregiving" au "breadwining ». Idée que l'on retrouve chez les infirmières indiennes émigrées dans les pays du Golfe dont nous parle Marie Percot, expliquant que c'est notamment pour leurs enfants et leur bien-être matériel qu'elles acceptent d'en être séparées. 


\section{De l'importance des facteurs structurels} le concert des sociétés de consommation - après de nombreuses années d'autarcie et de rigueur économe -, notamment à la faveur de la contribution des émigrés et de leurs précieux transferts monétaires qui finira par jouer un "mauvais tour " à ceux-là mêmes sur lesquels repose en partie cette formidable métamorphose. Développement $\mathrm{du}$ pays qui annule en effet l'avantage des émigrés - comparativement à leurs compatriotes restés en Espagne - qui pouvaient, un mois l'an, faire montre des richesses accumulées au coût de nombreuses privations, au jour le jour, dans le pays d'accueil. L'ethos de la consommation en vogue en Espagne ne l'emportera pas chez les immigrés espagnols en France sur celui de l'épargne, de la frugalité, intériorisé avant même la migration. Peut-être le "consumérisme» n'a-t-il été, dans l'esprit des immigré(e)s, que différé, mais le résultat est là : à la consommation ostentatoire des uns (les Espagnol(e)s en Espagne) s'oppose la sobriété des pratiques de consommation des autres (les immigré(e)s espagnol(e)s en France). Et, dans ce contexte, les pratiques dispendieuses des immigré(e)s de retour au pays pour les vacances n'impressionnent plus véritablement personne (Laura Oso Casas).

21 Le marché du travail canadien avec ses professions protégées et encadrées par des ordres professionnels, qui rend plus ardu l'accès des migrants hautement qualifiés à l'exercice de certains métiers en dépit, donc, de la possession des capitaux culturels adéquats (Krahn et al. 2001); la division sexuelle du travail rigide qui n'admet pas l'accès massif des femmes canadiennes à certaines professions techniques tenues pour masculines, ce qui rend d'autant plus difficile l'accès des femmes migrantes à ces mêmes emplois bien qu'elles soient issues d'un pays promouvant l'éducation des femmes et leur accès aux emplois considérés comme "masculins » à l'Ouest (Mihaela Nedelcu). Partout d'ailleurs, des États-Unis aux pays du Golfe, en passant par la France cette même division sexuelle du travail, qui traverse les époques et confine les femmes dans les emplois considérés comme peu qualifiés, par exemple les emplois dits de reproduction sociale : métiers du care, du nettoyage, des services sexuels.

Autre exemple de l'influence des facteurs structurels, les politiques migratoires, de fermeture des frontières, voire de production de nouvelles frontières (Richard, 2004), et de criminalisation de certaines formes de migration qui contribuent à produire de l'informalité et de l'illégalité (Morice, 2002) et qui influent, par exemple, sur le développement de la traite (Rutvica Andrijasevic).

Yen le Espiritu, précise l'importance de l'histoire coloniale des Philippines dans la configuration des flux migratoires de femmes infirmières de ce pays vers les États-Unis. Elle intègre celle-ci dans sa lecture critique du transnational qui met l'accent sur les structures globales de l'inégalité: "Moving beyond the telos of immigrant settlement, assimilation, and citizenship and the myth of «immigrant America ", a critical transnational 
perspective conceptualizes immigration not as a site for assessing acceptability of the immigrants, but as a site for understanding the racialized economic, cultural and political foundations of the United States». Le colonialisme américain dans ce pays a bien contribué à produire/susciter des opportunités sexuées et racialisées de migration. L'auteure nous rappelle également que les bordels philippins fréquentés par les Américains ont donné des femmes philippines une image hyper sexualisée dont sont encore victimes, d'une certaine façon, celles qui migrent de nos jours aux États-Unis. Par ailleurs, et plus généralement, une partie de la mobilité des femmes est elle-même structurellement et intimement liée aux différentes formes de mobilité masculines. Tourisme, mobilité des hommes d'affaires, de militaires, engagés dans des guerres ou dans des forces de "maintien de la paix », créent une demande de services (de reproduction sociale, sexuels). Citons pour exemple, la mobilité des managers qui implique celle du personnel de service visant à décharger leurs conjointes des tâches domestiques et ingrates du travail de reproduction sociale.

Les facteurs structurels jouent donc aussi bien sur les motivations de départ, sur les caractéristiques ou la composition même des flux migratoires - en termes de sexe, d'ethnicité ou d'appartenance religieuse, de classe -, sur les difficultés d'accès des migrants qualifiés au marché du travail, sur l'appréhension subjective de la mobilité sociale des immigrés, etc. Mais si les migrants se trouvent placés au sein de hiérarchies de pouvoirs qu'ils/elles n'ont pas construites (classe, race, ethnicité, nationalité, genre...) et qui influent sur leurs pratiques et représentations sociales, ils n'en développent pas moins en regard de ces hiérarchies différentes formes d'action (agency) à partir de leurs positions respectives. Les conditions structurelles peuvent constituer des obstacles mais également des opportunités à saisir : "même ceux qui paraissent les plus démunis ont la capacité à mobiliser des ressources et à se ménager des espaces de contrôle » (Giddens, 1982 : 187, cité par Goss et Lindquist, 1995).

\section{Des femmes actrices de leur migration}

En coordonnant ce numéro, notre intention de sortir de l'ornière des thèmes récurrents, les plus souvent étudiés lorsqu'il est question du couple genre et migrationemplois du care, travail domestique, prostitution - n'a pu entièrement se concrétiser. Mais ces thèmes, largement relayés dans ces pages, le sont dans une perspective différente. Loin des poncifs et de la victimisation des femmes en migration, les figures de migrantes qui nous furent proposées et qui apparaissent dans les articles témoignent de leur hardiesse, de leurs résistances, de leurs ressources. De leur rôle pionnier, par exemple, dans la chaîne migratoire; de leur aptitude à réorienter leurs projets et parcours migratoires, telles les infirmières indiennes dans les pays du Golfe (Marie Percot). De leur capacité, à trouver des solutions palliatives plus ou moins temporaires face aux contraintes structurelles qui les affectent comme celle qui voit les femmes roumaines qualifiées au Canada se lancer dans l'entrepreneuriat ou adopter des stratégies de survie faute d'accès à des emplois correspondant à leurs qualifications (Mihaela Nedelcu). Des stratégies particulières qu'elles développent et qui permettent d'asseoir leurs projets d'épargne et de retour comme les domestiques espagnoles qui optent en France pour des emplois de concierges afin de réduire les frais de logement et concilier travail productif et reproductif ou celles, célibataires, qui se maintiennent dans des emplois de domestiques à demeure afin, là encore, de maximiser leur épargne 
(Laura Oso Casas). De leur aptitude à investir - différemment des hommes - des espaces géographiques et sociaux usant pour cela, s'il le faut, de la ruse telles les femmes commerçantes tunisiennes qui font des va-et-vient entre divers pays (Camille Schmoll). De leur capacité à se défendre, en se regroupant, en s'organisant, en formant des alliances comme au sein du réseau RESPECT, et ce, pour effectuer du lobbying auprès d'institutions européennes, telles les employées domestiques (Helen Schwenken). De leur connaissance, au moins parcellaire, des conditions de leur entrée dans la prostitution et de la maîtrise de leurs parcours migratoire (Rutvica Andrijasevic). De leur utilisation tactique ou stratégique de la législation et/ou des institutions telles les femmes roumaines qui trouvent à se marier sur le web - avec des compatriotes déjà installés au Canada - pour migrer à la faveur d'un regroupement familial facilitant ainsi leur insertion sur le marché du travail. Insertion rendue autrement difficile par l'insuffisance des qualifications requises ou la faible reconnaissance de certaines formations dans un domaine moins bien apprécié par le système canadien d'évaluation à points ; de la contraction d'unions avec des personnes étrangères à leur communauté d'origine, permettant d'obtenir ainsi un statut stable et de développer des stratégies d'accumulation du capital scolaire, comme les filles au pair polonaises en France dont nous parle Dominique Giabiconi, même si ces mises en couple ne semblent pas préméditées. Et toujours, dans le même ordre d'idée, l'utilisation stratégique de l'image de soi en jouant sur la rhétorique de la victimisation afin de bénéficier de la possibilité de se maintenir sur le sol italien, avantage conféré par la loi aux femmes reconnues victimes de la traite et qui en font la demande. Possibilité que les femmes en provenance des pays de l'Est, par exemple, mettent à profit. Sans omettre l'utilisation stratégique par les organisations de défense des femmes immigrées du cadre favorable au gender mainstreaming au sein des institutions européennes de Bruxelles (Helen Schwenken). Mais, et phénomène frappant dans des sociétés habituées à la migration des hommes, il nous faut constater qu'être femme peut finalement constituer ou devenir un atout dans la migration. Être femme offre une opportunité de migrer lorsque par ailleurs existent des obstacles à la mobilité des hommes

\section{Mobilité spatiale et mobilité sociale}

Les unions mixtes peuvent être la voie d'une promotion sociale pour des femmes migrantes. Tel est par exemple le cas des Polonaises filles au pair en France décrites par Dominique Giabiconi. L'auteur fait une analyse socio-démographique de deux types de couples impliquant des Polonais : ceux formés de deux conjoints polonais et les couples binationaux. Les femmes polonaises, plus que les hommes, contractent des unions binationales à la différence, d'ailleurs, de ce qu'il est généralement possible d'observer parmi les migrants en France. Des différences sont par ailleurs notables entre ces deux types de couples lorsque l'on considère le milieu de provenance des femmes (milieu rural/milieu urbain), voire l'écart d'âge entre les conjoints et leur propension à l'homogamie/hypergamie sociale. Désir de promotion et d'autonomisation y compris pour les femmes que l'on ne perçoit généralement que comme étant soumises à la "traite ", victimes, trompées, à la merci des réseaux criminels. Rutvica Andrijasevic suggère que pour ces femmes la migration, y compris le recours en connaissance de cause aux réseaux de la traite pour migrer, constitue une voie d'accès au marché du 
travail de l'UE, une manière de résister aux inégalités structurelles et de lutter pour transformer leur vie.

Mais la mobilité souhaitée, projetée par les migrants peut, comme nous l'avons indiqué, se trouver contrariée compte tenu des transformations observées dans le pays de départ. Laura Oso Casas nous rappelle que la mobilité sociale comporte deux dimensions - objective et subjective - et que celle des migrants ne se mesure pas à la seule aune de leur trajectoire (à divers moments de leur existence) mais également à celle des personnes restées au pays d'origine. Et certainement plus particulièrement à celle de "personnes repères ", membres de la famille proche n'ayant pu ou n'ayant pas voulu migrer.

Le désir de mobilité peut encore être contrarié par d'autres facteurs. La contribution de Mihaela Nedelcu met en scène un phénomène qui commence à être souvent relevé dans divers pays : celui de la mobilité sociale descendante - ou différée - de personnes disposant de capitaux culturels qu'elles ne peuvent monnayer ou convertir dès l'arrivée dans la société d'accueil (Riaño, 2003 ; Cardu et Sanschagrin, 2002 ; Khran et al, 2001). Les recherches sur la mobilité des personnes hautement qualifiées laissent apparaître le même schéma que celui observé dans les migrations de main-d'œuvre des années 1960-1970: l'invisibilité des femmes, l'accent mis sur les hommes. Ou encore la présence des femmes qui suivent les hommes comme le soulignent Eleonor Kofman et Parvati Raghuram (2002; Raghuram, 2004). Alors que les recherches féministes mettent l'accent sur la sphère de la reproduction pour parler du sort des femmes professionnelles et examinent « les stratégies de compensation » mises en œuvre pour combler le manque d'activité professionnelle: associations, actions caritatives, bénévolat, etc. (Yeoh et Khoo 1998), Mihaela Nedelcu examine, elle, les stratégies déployées par les professionnelles roumaines pour recouvrer un statut professionnel et une indépendance économique en dépit des multiples obstacles qu'elles affrontent au Canada.

29 L'absence de reconnaissance des qualifications professionnelles des femmes chinoises de Hongkong, issues des classes moyennes, immigrées au Canada (Man, 1995) génère leur déclassement social, voire une situation de dépendance économique nouvelle visà-vis de leur conjoint. En outre, ces femmes sont coupées des réseaux de parenté élargie qui contribuaient à les décharger des tâches domestiques dans leur pays d'origine. Elles se trouvent donc, dans la migration, confrontées à une surcharge du travail de reproduction d'autant que des facteurs structurels (taille des villes, inadéquation des transports...) accroissent leurs obligations telles que le fait de conduire les enfants. Les infirmières Philippines aux États-Unis dont nous parle Yen Le Espiritu soulignent, elles aussi, la surcharge du travail due à l'absence de soutien du réseau de parenté.

Pourtant, sa recherche rejoint les travaux relatifs aux migrations aux États-Unis montrant qu'en dépit d'inégalités sexuées inhérentes au marché du travail, les femmes, par rapport aux hommes, "gagnent» dans la migration, tandis que ces derniers perdent en statut social (Pessar, 1999: 63). C'est le cas des femmes qui connaissent une situation professionnelle stable et ont été des têtes de pont de la migration familiale comme les infirmières philippines en meilleure position que leurs homologues masculins pour trouver du travail dans une économie américaine marquée par la pénurie de personnel médical. Ce type de situation illustre la limite des approches, critiquées d'ailleurs par divers auteurs, visant à mesurer l'appartenance de classe en ne 
prenant en compte que la position professionnelle du mari (Garnsey, 1982; Laufer et Marry, Maruani, 2003).

31 Cependant au sein des couples de professionnels les femmes sont celles qui font souvent des compromis, subissent une rupture dans leur carrière et n'obtiennent pas dans la migration une renégociation des tâches reproductives à long terme. L'augmentation même du statut social des femmes ne signifie pas nécessairement une amélioration de leur position dans les rapports entre les sexes. L'exemple d'Elizabeth, philippine, membre du corps médical montre que le "renversement de rôles", l'intolérable perte de statut pour l'homme ne sauraient être durables, le couple retrouvant son équilibre dans le modèle du rapport qui lui était familier aux Philippines.

D'autres récits dans ce numéro pointent des changements lents mais visibles qui, sans les bousculer, remettent en question les certitudes des rapports hiérarchiques dans les familles et vont dans le sens d'une plus grande autonomisation des femmes (" empowerment ») même s'ils ne vont pas sans s'assortir de " coûts sociaux » importants (surcharge de travail, stigmatisation, éloignement et distanciation des enfants).

$\mathrm{Au}$ Kerala, le métier jadis méprisé d'infirmière commence à forcer ou susciter le respect grâce aux femmes qui émigrent dans les pays du Golfe pour l'exercer. Leur réputation sur le marché matrimonial en témoigne. Ces femmes, dont certaines sont des transnational mothers, cherchent à garder un contrôle en matière d'éducation des enfants et un certain regard sur le budget auquel elles contribuent en se positionnant différemment dans la hiérarchie de la famille élargie. Les jeunes femmes philippines aux États-Unis, quant à elles, choisissent de manière plus autonome leur conjoint. Cependant Marta Tienda et Karen Booth dans leur état des lieux sur la modification des rapports sociaux de sexe en migration constatent que les rapports dissymétriques demeurent intacts alors même que des dimensions spécifiques des inégalités liées au genre sont modifiées (1991: 69). Par exemple le fait d'être économiquement indépendantes et même chefs de famille ne transformera pas nécessairement les identités de mères et épouses qui seront seulement adaptées à la nouvelle situation (Pessar, 1984 ; Morokvasic 1987).

34 Dans différentes contributions, il est également question de compromis, d'arrangements, de réciprocité, pour sauver les apparences ou maintenir un statu quo. Elles rendent compte, non pas d'un rapport frontal, de conflits, de ruptures entre femmes migrantes et hommes de leur entourage mais plutôt de tactiques ou stratégies d'évitement, de neutralisation de ces potentielles oppositions. Notons déjà que la circulation des femmes tunisiennes et le départ des femmes indiennes du Kerala sont négociés ou impulsés par la famille, voire, dans le cas des migrantes roumaines, encadrée par un l'un de ses membres masculins.

Dans un contexte où l'incursion et l'activité professionnelle des femmes migrantes dans l'espace public sont mal vues - les Tunisiennes circulant entre Naples et Sousse -, elles peuvent en venir à se servir des hommes comme alibis pour être mobiles. La difficulté des hommes à obtenir des visas pour l'Italie leur permet de justifier leur propre mise en mobilité. Ces femmes se cachent, ou cachent leur motivation derrière ce prétexte, comme si elles cherchaient, coûte que coûte, à préserver l'état des rapports sociaux de sexe ou, tout du moins, leur apparence. On peut encore avancer l'idée que les femmes se servent des hommes comme prête-noms pour leur entreprise commerciale, autre façon de ne pas minorer la position de l'homme, de ne pas lui faire, publiquement, 
perdre la face. L'exemple des infirmières du Kerala montre qu'elles ne remettent pas nécessairement en cause la dot - que leur émigration contribue indirectement à financer -, mais qu'elles l'utilisent à leur profit. Les pratiques traditionnelles deviennent des outils mêmes de transformation des rapports sociaux entre les sexes.

Des travaux ont montré les efforts déployés par les femmes en migration pour atténuer les coûts sociaux du transnational motherhood. Ils ont mis en exergue que la temporalité des circulations migratoires pouvait être subordonnée aux exigences de la vie familiale. Ainsi en est-il par exemple du système de rotation autogérée mis en œuvre par certaines femmes polonaises travaillant dans le service domestique en Allemagne: généralement jamais plus de cinq par groupe, elles alternent de courtes périodes de travail en Allemagne et des périodes plus longues en Pologne. Ainsi, tout en travaillant par moments chez des employeurs allemands, elles demeurent disponibles et présentes au sein de leur famille. Si la rotation est dictée par des obligations familiales en Pologne, ce quasi-système de partage de l'emploi entre employées domestiques les protège d'une dépendance moins accentuée vis-à-vis de leur employeur. Il tend aussi à minimiser les risques, dont celui de se retrouver dans une situation illégale sur le territoire allemand (Morokvasic, 1999).

À l'instar des Polonaises en Allemagne ou en Belgique décrites par Irek (1998), Kuzma (2003), Morokvasic (2004), les femmes circulantes tunisiennes s'organisent pour éviter des séjours prolongés à Naples et se garder des risques de ruptures familiales, même si elles se reposent parfois sur l'aide d'autres femmes qui les assistent dans les tâches domestiques. Circuler, être mobile pour ne pas migrer définitivement et se couper de son réseau familial, prend ici toute sa mesure.

Notons des formes originales - ou tout du moins rarement relatées - de prise en charge des travaux de reproduction dans un cadre transnational, qui ne sont pas sans rappeler le global care chain (Hochschild 2000). Des grands-parents roumains des deux sexes, la précision est d'importance, alternent leur présence chez leurs enfants installés au Canada pour s'occuper de leur progéniture. Notons encore la migration vers les pays du Golfe de jeunes femmes indiennes venant s'occuper, à bas prix, des enfants de leurs compatriotes infirmières qui, pour leur part, travaillent dans des institutions prodiguant des soins aux personnes (les hôpitaux). Ces cas illustrent la proposition d'extension du Global care Chain que Yeates (2004) a développée à propos de la situation des infirmières en Irlande.

39 Si la migration des femmes et les liens transnationaux peuvent être empowering (émancipateurs), il n'est nullement question ici de nier ou de minimiser les violences faites aux femmes, l'exploitation sexuée multiforme (traite des femmes, prostitution...) dans la migration ou en amont de celle-ci qui peut emprunter différents canaux (réseaux criminels, Internet...). Même si les auteurs de contributions (Andrijasevic, Boukhobza), mettent en garde contre des généralisations consistant à victimiser les femmes sans reconnaître la pluralité de leurs parcours, il faut reconnaître que des femmes peuvent être trompées sur le réel motif de leur migration et conduites à exercer une activité contre leur gré (prostitution ou autre).

\section{La difficulté d'articuler sexe/classe et ethnicité}

La contribution de Christian Poiret rend compte de la difficulté à penser conjointement des rapports de domination de sexe, de classe et ethniques. Elle porte sur l'articulation 
des rapports sociaux de sexe, de classe et interethniques dans les champs académique, militant et juridique nord-américains et a vocation à stimuler la réflexion sur la mise en pratique de cette articulation dans le cas français. L'auteur présente le cheminement ayant mené les Black Feminists à poser la question de l'intersectionalité de ces rapports. S'appuyant sur les enseignements qu'il est possible de tirer de la jurisprudence nordaméricaine relative à l'application des lois anti-discriminatoires et sur sa critique, l'auteur met déjà en garde contre le danger qui consiste à appréhender les causes des discriminations non plus dans les relations sociales mais dans les caractéristiques mêmes des individus, processus aboutissant à essentialiser les catégories de l'altérité. Les caractéristiques, voire l'une d'entre elles puisque plusieurs arrêts émanant de cours nord-américaines n'ont pris en compte qu'une seule oppression, la discrimination sexuée ou raciste chez des plaignantes se déclarant victimes de ces deux discriminations. Ces personnes seront reconnues discriminées sur la base de leur appartenance au sexe féminin (et ici l'appartenance à des groupes minoritaires disparaît) ou à "raison de la "race " " (c'est ici l'appartenance sexuée qui est invisibilisée), et ce en référence à un groupe dominant composé d'hommes, sans handicap, etc. Cette critique n'est pas sans rappeler celle parfois formulée à l'encontre des approches postmodernes défendant cette articulation. Ériger les Black women et les femmes du Tiers-Monde en figure d'altérité au sein des femmes, cette fois-ci, conforte finalement les femmes blanches sur leur piédestal. Comme le rappelle Eleni Varikas (1993: 78).

«Marques de différence, elles sont le plus souvent intégrées dans le discours féministe en fonction de leur capacité à témoigner de ce qui excède l'expérience des femmes ou des féministes "blanches", de ce qui peut permettre à ces dernières de se comprendre plutôt que de les comprendre. Ce traitement instrumental des expériences différentes des femmes "de la périphérie » ou des «marges", et la polarité dichotomique que celui-ci entretient (Occident/non-Occident), risque encore une fois d'homogénéiser l'autre, perçue prioritairement à travers ses différences par rapport à l'une. [...]. Ainsi, le statut différent de ces expériences féminines n'est reconnu, et l'unicité de la catégorie femmes n'est mise en cause qu'au prix d'une unicité reconstruite de la catégorie femmes de couleur ou femmes du Tiers Monde. Si ces dernières deviennent visibles, cette visibilité implique souvent l'absorption des différences qui existent parmi elles, et donc une nouvelle construction de stéréotypes d'altérité ».

41 La contribution de Noria Boukhobza attire d'ailleurs notre attention sur le danger de réification des immigré(e)s perçus comme un Autre. Les représentations sociales relatives aux femmes et aux filles, aux hommes et garçons d'origine maghrébine en France sont-elles sexuées? Les voit-on de la même façon? Au même moment ? Les filles naissent après les garçons, conclut-elle. Formule rendant compte d'un double phénomène. L'imagerie sociale a réinventé et figé l'ordre des naissances, la place des filles et des garçons au sein des familles maghrébines : ainsi les filles ne sauraient être que des cadettes dans l'ombre d'un grand frère; les filles, en outre, n'acquièrent une visibilité médiatique que postérieurement aux garçons, suite aux violences sexuelles perpétrées dans les quartiers ou à l'occasion de la médiatisation des affaires du voile (années 1990 puis années 2000). Ordre des naissances physique et symbolique, parfois virtuel qui pose avec acuité la question de l'articulation sphère privée/sphère publique. Dans quelle mesure le regard réifiant posé sur les familles maghrébines façonne-t-il celui jeté sur ces populations dans l'espace public? Ou est-ce l'inverse? Quoi qu'il en 
soit, c'est un plaidoyer pour la prise en compte et la reconnaissance de la multiplicité des parcours des femmes, de leurs caractéristiques que présente l'auteur.

Enfin, une autre question posée dans la contribution de Christian Poiret est la difficulté à déterminer, dans le champ militant, une priorité en termes revendicatifs au sein d'une coalition d'organisations identitaires. Sur quel type d'oppression ou de domination les coalitions doivent-elles finalement mettre l'accent? L'article d'Helen Schwenken explore justement cette difficulté d'articuler ces trois rapports de domination plus spécifiquement dans les luttes ou mobilisations collectives. Se basant sur l'exemple du réseau RESPECT, voué, comme nous l'avons signalé, à défendre les intérêts d'employés domestiques en effectuant du lobbying auprès des institutions européennes, il montre - ce qui est intéressant - que ce n'est pas nécessairement le thème en vogue, celui supposé avoir le plus de résonance, d'impact politique (par exemple la question de la traite des femmes) qui est mobilisé par les organisations pour défendre les droits de leurs membres ou leurs revendications.

\section{Conclusion}

43 Femmes, genre, migration et mobilités, marque le passage de l'étude des femmes en migration à celle du genre et de la migration, de la migration entendue dans son acception classique (le fait de se mouvoir d'un espace d'origine à un espace d'accueil et de s'y installer pour une durée plus ou moins longue) à des formes différentes, plus variées de déplacements : migrations, circulations, va-et-vient que nous englobons sous le terme «mobilités ». Mobilité faisant également référence au passage d'un statut ou d'une position sociale à une autre dans la hiérarchisation sociale, que nous pouvons rapprocher des changements/reconfigurations des rapports sociaux de sexe.

À la lecture des contributions publiées dans ce numéro, quelques questions se dégagent :

- Les réseaux migratoires fonctionnent-ils différemment pour les hommes et les femmes ? Les politiques migratoires supposées neutres sont-elles genrées et quels effets différenciés ontelles sur les hommes et les femmes ? Peut-on parler de « savoir-circuler », de savoir faire du commerce ou manières de s'organiser, de défendre ses droits, faire des alliances, s'inscrire dans des revendications et luttes etc. typiquement «féminins» (ou "masculins») sans inclure les hommes? Peut-on déduire des études portant sur les seules femmes des réponses à ces questions?

- En confrontant les recherches menées en Europe et aux États-Unis sur le transnational on a noté, dans ce dernier cas, la propension des femmes à s'investir dans le local plutôt que dans le transnational, à l'inverse des hommes. Les recherches présentées ici pointent, par contre, l'implication des femmes migrantes dans les activités, réseaux et circulations transnationales et ce, parfois plus que les hommes. À quoi renvoient ces différences d'observations? Les mobilités transnationales circulatoires seraient-elles, par ailleurs, une phase passagère, temporaire ou transitoire vers la constitution de communautés transnationales?

- La question des éventuels changements induits par la migration - des hommes, des femmes séparément ou conjointement - dans les rapports sociaux de sexe (autonomisation, accentuation des pouvoirs, etc.) a été largement discutée. Il est visible que les effets de la migration ne sont pas nécessairement empowering ou, tout du moins, ne se traduisent pas nécessairement par une modification des rapports sociaux de sexe au sein du couple ou de la 
famille. Si la question des éventuels changements découlant de la migration est au demeurant classique, celle du « comment » de ces transformations est restée, pour sa part, en suspens. Or, il semble à la lecture de diverses contributions que la négociation soit privilégiée à l'affrontement entre les sexes au cours des diverses phases de la migration des femmes (décision du départ, capitalisation des acquis de la migration, etc.).

- Acteurs et actrices vulnérables, les migrant(e)s sont amené(e)s à instrumentaliser leur image de victime/se servir à leur avantage de la rhétorique de la victimisation pour défendre leurs intérêts et obtenir des droits, compte tenu de la résonance et de l'impact politiques à court terme Mais il semblerait - et d'autres recherches sur la question sont nécessaires - qu'à long terme, à l'inverse, ces personnes puissent refuser cette image victimisante alors même qu'elle aurait pu servir les intérêts défendus (face aux et grâce aux organisations internationales par exemple). Et ce, lorsqu'elle est perçue comme antinomique avec l'image de soi que l'on entend donner: une image d'acteurs ou d'actrices. Idée qui renvoie à une phrase de Ghandi citée dans un article: «ce que vous faites pour nous, sans nous, est contre nous ».

\section{BIBLIOGRAPHIE}

ANTHIAS Floya, LAZARIDIS Gabriella (2000) Introduction : Women on the Move in Southern Europe in F. Anthias \& G. Lazaridis (ed.), Gender and Migration in Southern Europe: Women on the Move, Oxford ; New York, Berg, pp. 1-13.

CARDU Hélène, SANSCHAGRIN Mélanie (2002) Les femmes et la migration : les représentations identitaires et les stratégies devant les obstacles à l'insertion socioprofessionnelle à Québec, Recherches féministes, 15 (2), http://www.erudit.org/revue/rf/2002/v15/n2/006512ar.html

Cahier du CEDREF (2003) Genre, travail et Migrations en Europe.

Cahier du CEDREF (2000) femmes en migrations, $n^{\circ}$ 8-9.

CERTEAU Michel de (1990) L'invention du quotidien. 1. Arts de faire, coll. Folio Essais, Paris, Gallimard, $2^{\text {ème }}$ édition, $349 \mathrm{p}$.

CHANT Sylvia (Ed.) (1992) Gender and Migration in Developing Countries, London and New York, Bellhaven Press.

COMMISSARIAT GÉNÉRAL DU PLAN (2002) Immigration, marché du travail, intégration, Paris, La documentation Française, 409 p.

DORAI Kamel, HILY Marie-Antoinette et MA MUNG Emmanuel (1998) La circulation migratoire. Bilan des travaux, Migrations Etudes, ${ }^{\circ} 84$, décembre, $12 \mathrm{p}$.

EHRENREICH Barbara, HOCHSCHILD Arlie Russell (2003) Global Woman : Nannies, Maids, and Sex Workers in the New Economy, New York, Metropolitan Books, 328 p.

FERREE Myra Marx (1979) Employment without liberation : Cuban women in the United States, Social Science Quarterly, 80 (1), pp. 35-50. 
GARNSEY Elisabeth (1982) Women's work and theories of class and stratification in A. Giddens, D. Held (eds.), Classes, power, and conflict. Classical and contemporary debates, Berkeley, Los Angeles, University of California Press, pp. 425-445.

GIDDENS Anthony (1982) Profiles and Critiques in Social Theory, Berkeley, University of California Press.

GLICK SCHILLER, Nina, BASCH Linda, SZANTON-BLANC Cristina (1992), Towards a Transnational perspectives on Migration, New York, News York Academy of Sciences.

GLICK SCHILLER Nina et Georges Eugene FOURON (2001), Georges Woke Up Laughing : Long-Distance Nationalism and the Search for Home, Durham, NC : Duke University Press.

GOSS Jon D., LINDQUIST Bruce (1995) Conceptualizing international labor migration : a structuration perspective, International Migration Review, XXIX (2), Summer, pp. 317-351.

HEYZER Noyleen, LYCKLAMA a NIJEHOLT Geertje et WEERAKOON Nedra (1992) (dir.) The Trade in Domestic Workers. Causes, Mechanisms and Consequences of International Migration, London/New Jersey, Zed Books Ltd ; APCD : Kuala Lumpur, 247 p.

HILY Marie-Antoinette et MA MUNG Emmanuel (sous la dir.de) (2002) Catégories et lieux des circulations migratoires, Rapport de recherche pour la MIRE, $235 \mathrm{p}$.

HOCHSCHILD Arlie Russell (2000) Global care chains and emotional surplus value, in W. Hutton and A. Giddens (eds.), On the Edge : Living with Global Capitalism, London, Jonathan Cape.

HONDAGNEU-SOTELO Pierrette, AVILA Ernestine (1997) «I'm here, but I'm there ». The meanings of Latina Transnational Motherhood, Gender and Society, 11 (5), pp. 548-571.

Hommes et Migrations (2004) Femmes contre la violence, $n^{\circ}$ 1248, mars-avril.

HUANG Shirlena, YEOH Brenda S. A. (2003) The difference gender makes : State policy and contract migrant workers in Singapore, Asian and Pacific Migration Journal, 12 (1-2), pp. 75-97.

HUGO G. (1999) Gender and Migrations in Asian Countries, Liège, International Union for the Scientific Study of Population.

IREK Margolzata. (1998) Der Schmugglerzug. Warschau-Berlin. Warschau. Materialien einer Feldforschung, Berlin, Das Arabische Buch

KELSON A. Gregory, DELAET L. Debra (1999) Gender and Immigration, London : Macmillan

KIVISTO P. (2001) Theorizing transnational immigration : A critical review of current efforts. Ethnic and Racial Studies, 24 (4), pp. 549-577.

KOFMAN Eleonore (2004) Family-Related Migration : A Critical Review of European Studies, Journal of Ethnic and Migration Studies 30 (2), pp. 243-262.

KOFMAN Eleonore, PHIZACKLEA Annie, RAGHURAM Parvati, SALES Rosemary (2000) Gender and International Migration in Europe. London, Routledge, $234 \mathrm{p}$.

KOFMAN E., PHIZACKLEA Annie et al. (2000) Gender and migration theory, in E. KOFMAN Gender and International Migration in Europe, London, Routledge, pp. 21-43.

KOFMAN Eleonor (1999) Birds of passage a decade later : gender and immigration in the European Union, International Migration Review, 33 (3), pp. 269-299.

LAUFER, J., MARRY C.,MARUANI M. (2003) Introduction in J. Laufer, C. Marry, M. Maruani, Le travail du genre. Les sciences sociales du travail à l'épreuve des différences de sexe, Paris, La Découverte/ MAGE, pp. 7-18. 
LUTZ H. (1998) The legacy of migration : immigrant mothers and daughters and the process of intergenerational transmission, in M. Chamberlain (ed.), Carribean migration : globalised identities, London and New York, Routledge, pp. 95-108.

MAN Guida (1995) The experience of women Chinese immigrant families : an inquiry into institutional and organizational processes, Asian and Pacific Migration Journal, 4 (2-3), pp. 303-326.

MATHIEU Nicole (2000) Sexe et Genre, in H. Hirata, F. Laborie, H. Le Doaré et Senotier, D. (dir.) Dictionnaire critique du féminisme, Paris, Presses Universitaires de France, pp.191-200.

MICHALON Bénédicte (2003) « Circuler entre Roumanie et Allemagne ». Les Saxons de transylvanie, de l'émigration ethnique au va-et-vient, Balkanologie, 7 (1), pp. 19-42.

MIGRATIONS SOCIÉTÉ (1997) Femmes immigrées en France et en europe, 9 (2), juillet août MORICE Alain (2002) Retour de l'immigré utile, Manière de Voir 62, Le Monde diplomatique, marsavril, pp. 43-46.

MOROKVASIC Mirjana (1976) L'immigration féminine en France : l'état de la question, L'année sociologique, 26 (2), pp. 563-575.

MOROKVASIC Mirjana (2004) 'Settled in mobility’: engendering post-wall migration in Europe, Feminist Review, 'labour migrations : women in the move', 77, pp. 7-25.

MOROKVASIC Mirjana (2003) Transnational mobility and gender : a view from post-wall Europe, in Morokvasic, Mirjana, Erel, Umut and Shinozaki, Kyoko : Crossing Borders and Shifting Boundaries. Vol. I : Gender on the Move. Opladen : Leske + Budrich, pp. 101-133.

MOROKVASIC Mirjana (1999) La mobilité transnationale comme ressource : le cas des migrants de l'Europe de l'Est, Cultures et Conflits, (32), pp. 105-122.

MOROKVASIC Mirjana (1987) Emigration und danach. Jugoslawische Frauen in Westeuropa. Frankfurt/ Main : Stroemfeld-Roter Stern, 254 p.

MOROKVASIC Mirjana (1984) Birds of passage are also women, International Migration Review, "Women in Migration", 18 (68).

MOULIER Yann et SILBERMAN Roxane (1982) La montée de l'activité des femmes étrangères en France : une tendance qui ira s'accentuant, Travail et Emploi, n²12, juin-juillet, pp. 61-81.

OGAYA Chiho (2004) Social discourses on Filipino women migrants, Feminist Review, 'labour migrations : women in the move', 77, pp. 180-182.

PAPERMAN Patricia et PIERROT (1978) Le Travail ambigu, Cerfise, Cordes, n74-78, Paris, 283 p.

PARREÑASRhacel Salazar (2001) Servants of globalization: Women, migration and domestic work. Stanford, CA : Stanford University Press.

PERALDI Michel, BETAIEB Ajer, MANRY Véronique (2001) L'esprit de bazar. Mobilités transnationales maghrébines et sociétés métropolitaines. Les routes d'Istanbul, in Michel Peraldi (ed.) Cabas et containers. Activités marchandes informelles et réseaux migrants transfrontaliers, Paris : Maisonneuve et Larose, pp. 329-361.

PESSAR Patricia R (2001) Women's political Consciousness and Empowerment in Local, National and Transnational Contexts : Guatemalan Refugees and Returnees. Identities : Global Studies in Culter and Power, 7, pp. 461-500.

PESSAR Patricia R (1999) The role of gender, households, and social networks in the migration process : A review and appraisal, in C. Hirschman, P. Kasinitz and J. DeWind (eds) The Handbook of International Migration, New York, Russell Sage Foundation, pp. 53-70. 
PESSAR, Patricia (1984) The linkage between the household and workplace of Domican U.S. women in the U.S., International Migration Review, 18 (4), Winter, pp. 1188-1211.

PHIZACKLEA Annie (1998) Migration and Globalization : a Feminist Perspective, in H. Lutz and K. Koser (eds.), The New Migration in Europe. Social Constructions and Social Realities, London, Macmillan, pp. 21-38.

PHIZACKLEA Annie (1983) One Way Ticket. Migration and Female Labour, London : Routledge and Kegan Paul, 162 p.

PIORE Michael (1979) Birds of Passage : Migrant Labour and Industrial Societies. Cambridge, Cambridge University Press, 229 p.

PORTES A., GUARNIZO L. E., LANDOLT P. (1999) The study of transnationalism : pitfalls and promise of an emergent research field, Ethnic and Racial studies, 22 (2).

PORTES Alejandro (1999) Conclusion : toward a new world - the origins and effects of transnational activities, Ethnic and Racial studies, 22 (2), pp. 217-237. PRIBILSKY Jason (2000) Más Modernos y Progresivos : Ecuadorian Transnational Fathers and the Gendered Subjectivities of Migrant Identity. Paper delivered at the 99th Annual Meeting of the American Anthropological Association. San Francisco, CA, November 16-19.

PRIBILSKY Jason (2004) 'Aprendemos A Convivir': Conjugal Relations, Co-parenting, and Family Life Among Ecuadorian Transnational Migrants in New York and The Ecuadorian Andes, Global Networks, 4 (3), July.

RAGHURAM Parvati (2004) The difference that skills make : gender, family migration strategies and regulated labour markets, Journal of Ethnic and Migration studies, 30 (2), pp. 303-321.

REMI (1999) Emploi, Genre et Migration, 15 (2).

RIAÑO, Yvonne (2003) Migration of Skilled Latin American Women to Switzerland and Their Struggle for Integration, in Mutsuo Yamada Ed., Emigración Lationoamericana : Comparación Interegional entre América el Norte, Europa et Japón. Population Movement in the Modern World VII. JCAS Symposium Series 19. The Japan Centre for Area Studies, National Museum of Ethnology, Osaka. http://www.giub.unibe.ch/sg/mitarbeiterinnen/Yvonne/The \% 20Struggle \% 20of \% 20LA \% 20Women \% 20for \% 20Integration.pdf

SALAF Janet (1997) The gendered social organisation of migration as work, Asian and Pacific Migration Journal, 6 (3-4), pp. 295-316.

SHAH Nasra M. (2004) Gender and labour migration to the Gulf countries, Feminist Review, 'labour migrations : women in the move', 77, pp. 183-185.

TAPIA de Stéphane (1994) l'émigration turque : circulation migratoire et diasporas, l'Espace Géographique, 23 (1), pp. 19-28.

TAPINOS Georges (1990) Development assistance strategies and emigration pressure in Europe and Africa (Working paper ${ }^{\circ} 56$ ), Washington D.C : Commission for the Study of International Migration and Cooperative Economic Development.

TARRIUS Alain (1992) Les fourmis d'Europe. Migrants riches, migrants pauvres et nouvelles villes internationales, Paris, L'Harmattan.

TARRIUS Alain (avec Lamia MISSAOUI) (2000) Les nouveaux cosmopolitismes. Mobilités, identités, territoires, Editions de l'Aube, $265 \mathrm{p}$.

TIENDA Martha, BOOTH Karen (1991) Social Change, International Sociology, 6 (1), pp.51-72 
TRUONG Thanh-Dam (1996) Gender, international migration and social reproduction :

implications for theory, policy research and networking, Asian and Pacific Migration Journal, 3 (1), pp. 27-51.

VARIKAS Eleni (1993) Féminisme, modernité, postmodernisme : pour un dialogue des deux côtés de l'océan, Futur Antérieur, Supplément, « Féminismes au Présent », pp. 59-84.

WILLIS Katie \& Brenda YEOH (eds.) (2000) Gender and Migration, Edward Elgar, 'International Studies in Migration' series.

WILLIS Katie, Brenda YEOH (eds.) (2000) Introduction in K. Willis, B. Yeoh (2000) (eds.) Gender and Migration, Edward Elgar, 'International Studies in Migration' series, pp. xi -xxii.

YEATES Nicola (2004) A dialogue with 'global care chain' analysis : nurse migration in the Irish context, Feminist Review, 'labour migrations : women in the move', 77, pp. 79-95.

YUVAL-DAVIS Nira (1997) Gender and Nation, London, Sage.

ZAIDMAN Claude (avec la collaboration de Prisca BACHELET) (2003) Introduction, Genre, travail et migrations en Europe, Collection des Cahiers du CEDREF, Publications Paris 7, pp.10-22.

ZLOTNIK Hania (2003) The global dimensions of female migration, Migration Information Source, http://www.migrationinformation.org/Feature/display.cfm ? ID = 109

\section{NOTES}

1. Womenandchildren de Nira Yuval-Davis (1997) qui utilise cette expression pour montrer que les femmes sont toujours attachées aux enfants.

\section{INDEX}

Mots-clés : femmes, genre, mobilité sociale, mobilité, rapports sociaux, sexe

\section{AUTEURS}

\section{CHRISTINE CATARINO}

Chercheure post-doc, associée au Laboratoire d'analyse des systèmes politiques - LASP, Université de Paris X - Nanterre

\section{MIRJANA MOROKVASIC}

Directrice de recherche au CNRS, Laboratoire d'analyse des systèmes politiques - LASP, Université de Paris X - Nanterre 